\title{
Primary Gastric Yolk Sac Tumour
}

"Asim Qureshi, ${ }^{1}$ Mansour Al-Moundhri, ${ }^{2}$ Maha Al-Shaibi, ${ }^{3}$ Ibrahim Al-Haddabi, ${ }^{1}$ Alok Mittal ${ }^{4}$

$$
\text { عاصم قرشي، منصور المنذري، مها الشعيبة، إبراهيم الهدابي، الوك ميتال }
$$

ABSTRACT: Primary gastric yolk tumours are extremely rare. We report a 52-year-old male who presented to the Sultan Qaboos University Hospital, Muscat, Oman, in 2017 after having undergone a gastrectomy abroad due to a suspected poorly-differentiated adenocarcinoma. The patient subsequently returned to Oman to receive chemotherapy. However, while undergoing chemotherapy, an abdominal computed tomography scan revealed a lobulated mesenteric mass. Microscopic examination of the resected lesion confirmed a diagnosis of a yolk sac tumour. The mass was diffusely positive for $\alpha$-fetoprotein (AFP) and a gastric carcinoma stain was negative. Gastrectomy slides from the patient's previous surgery were examined retrospectively. The morphology was typical for a yolk sac tumour and was negative for epithelial markers. An AFP stain showed diffuse immunoreactivity. Thus, the patient was deemed to have had a primary gastric yolk sac tumour which had later metastasised to the mesocolon. Germ cell tumour protocols were initiated and the patient responded well to treatment.

Keywords: Yolk Sac Tumor; Germ Cell Tumor; Gastrectomy; Metastasis; Diagnostic Errors; Case Report; Oman.

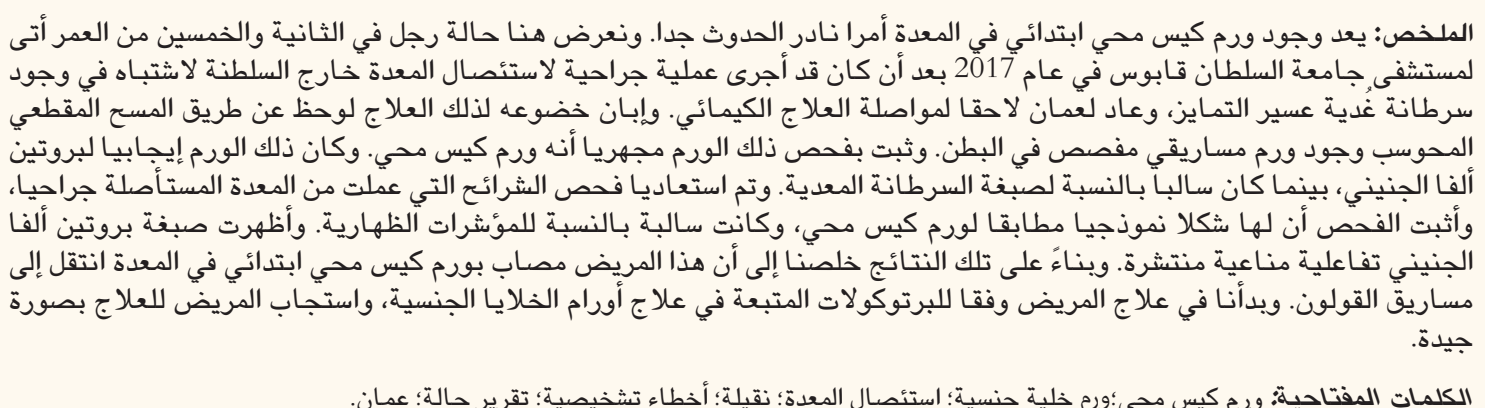

W HILE MOST GERM CELL TUMOURS OCCUR in the gonads, less common sites include the retroperitoneum, mediastinum and coccygeal region. ${ }^{1-4}$ Primary visceral germ cell tumours are exceedingly rare, with even fewer cases of primary gastric germ cell tumours. ${ }^{2,5}$ To the best of the authors' knowledge, this is the first case reported from Oman.

\section{Case Report}

A 52-year-old male presented to the oncology clinic of the Sultan Qaboos University Hospital, Muscat, Oman, in 2017. He had been diagnosed with stage IIIB gastric cancer in another country in 2016. At that time, contrastenhanced axial and coronal computed tomography (CT) scans taken at the level of the stomach showed a circumferential irregular enhanced wall thickening of the antropyloric region of the stomach, with mild luminal narrowing [Figure 1]. The patient underwent an open distal gastrectomy, omentectomy and lymph node dissection. The histopathology results were reported to indicate a poorly- differentiated adenocarcinoma involving the body and antrum of the stomach, with four out of six lymph nodes positive for metastatic carcinoma. The final diagnosis was of a poorly-differentiated adenocarcinoma (pT4aN3a). He was therefore advised to undergo six cycles of chemotherapy.

After beginning chemotherapy abroad, the patient travelled back to Oman and presented to the Sultan Qaboos University Hospital where treatment was completed over the following six months. Endoscopies and CT scans performed at regular intervals showed no signs of disease recurrence and the patient remained asymptomatic during this period. However, 16 months after the initial diagnosis, follow-up axial and sagittal contrastenhanced CT scans of the abdomen revealed a large heterogeneously-enhancing soft tissue nodule in the transverse mesocolon [Figure 2]. There was no evidence of any other disease sites in the chest or abdomen.

The mesenteric mass was biopsied under imaging guidance. A microscopic examination showed an infiltrating malignant epithelial tumour consistent with a 

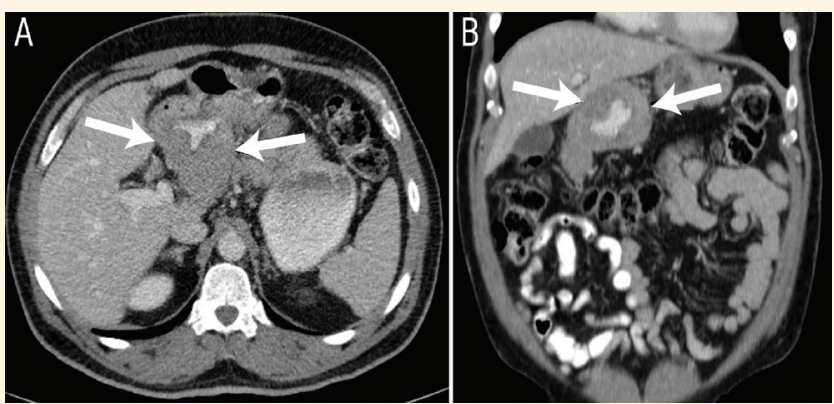

Figure 1: Contrast-enhanced (A) axial and (B) coronal computed tomography scans of a 52-year-old male taken at the level of the stomach showing circumferential irregular enhanced wall thickening (arrows) of the antropyloric region of the stomach, with mild luminal narrowing.
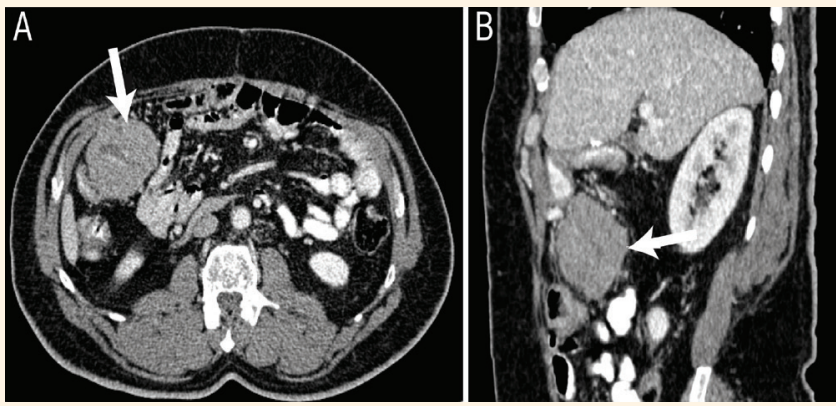

Figure 2: Follow-up (A) axial and (B) sagittal contrast-enhanced computed tomography scans of the abdomen of a 52-yearold male showing a large heterogeneously-enhancing soft tissue nodule (arrows) in the transverse mesocolon.

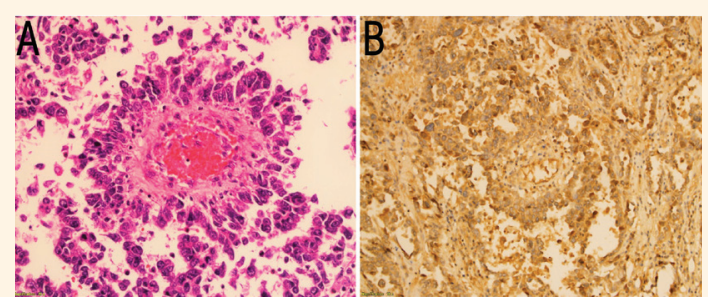

Figure 3: A: Haematoxylin and eosin stain at x20 magnification of a mesenteric mass showing tumour cells arranged around a blood vessel and forming Schiller-Duval bodies. B: Immunohistochemistry stain at x10 magnification showing diffuse positivity for $\alpha$-fetoprotein.

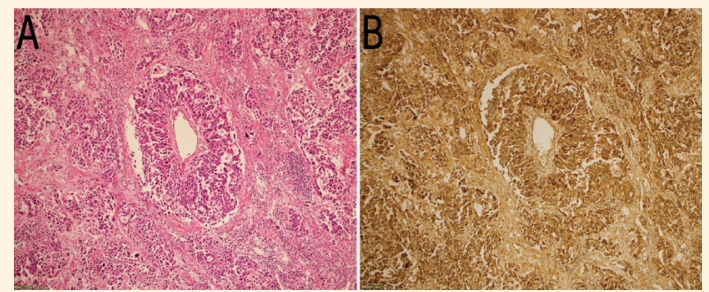

Figure 4: A: Haematoxylin and eosin stain at x10 magnification of a gastrectomy sample showing classic SchillerDuval bodies. B: Immunohistochemistry stain at x10 magnification showing diffuse $\alpha$-fetoprotein positivity.

poorly-differentiated carcinoma. However, a definite diagnosis could not be made due to the poor quality of the material and the lack of tissue available for immunohistochemical analysis. As there was no evidence of disease elsewhere in the body, the decision was made to remove the mass since it was highly unlikely to be a metastasis from a primary tumour. The patient underwent an explorative diagnostic laparoscopy and removal of the mass. During the procedure, the mass measured $8 \times 5 \mathrm{~cm}$, appeared well-circumscribed and encapsulated and was located in the right upper quadrant of the abdomen. It was densely adherent to the right lateral abdominal wall. The capsule of the mass was kept intact throughout the procedure and easily removed via a small midline laparotomy incision.

Following the procedure, the patient recovered well and was discharged without any complications on the third postoperative day. Histological sections of the mass revealed tumour cells arranged in a loose reticular meshwork around blood vessels, forming Schiller-Duval bodies [Figure 3A]. In some areas, the tumour cells were arranged in papillary structures. There was diffuse immunohistochemical positivity for $\alpha$-fetoprotein (AFP) [Figure 3B]. In addition, cytokeratin (CK) AE1/AE3, epithelial membrane antigen and cluster of differentiation (CD)10 stains were positive, whereas CK7, CK20, CD56, caudal type homeobox 2, cyclin D1, thyroid transcription factor 1 , hepatocyte paraffin 1 , renal cell carcinoma and melanoma antigen 1 stains were negative.

The final histopathological diagnosis was of a metastatic yolk sac tumour. In light of this, the histopathology slides from the initial gastrectomy procedure were reviewed retrospectively. Sections of the gastric mass showed classic Schiller-Duval bodies [Figure 4A]. 
In addition, there was diffuse AFP positivity [Figure 4B]. Both the morphology of the cells and immunohistochemistry results were compatible with a yolk sac tumour. Based on these findings, it was advised that the patient undergo a CT brain scan and an ultrasound examination of the testes, both of which were normal. Serum levels of other tumour markers, including lactate dehydrogenase, AFP and $\beta$-human chorionic gonadotropin, were normal. The final diagnosis was of a primary yolk sac tumour of the stomach with metastatic disease to the mesentery. Four cycles of etoposide and cisplatin were recommended as treatment. At the time of writing, the patient was receiving chemotherapy and doing well.

\section{Discussion}

Yolk sac tumours are a type of primary gonadal malignancy; however, presentation as a primary gastric tumour is extremely rare. To the best of the authors' knowledge, only a few cases have been reported in the literature..$^{2,5}$ The current case represented a diagnostic dilemma as the patient was initially diagnosed elsewhere with a poorly-differentiated adenocarcinoma and underwent an open distal gastrectomy. However, a mesenteric mass was subsequently found which showed typical morphological characteristics of a yolk sac tumour, with no evidence of disease elsewhere in the body. A retrospective review of the histology slides of the materials from the previous surgery confirmed the diagnosis of a gastric yolk sac tumour which had metastasised to the transverse mesocolon.

Certain gastric carcinomas may demonstrate a morphology similar to that of yolk sac tumours. ${ }^{6}$ In these circumstances, the role of immunohistochemistry is crucial. Gastric carcinomas are CK7-positive and AFPnegative, while the reverse is true for yolk sac tumours. ${ }^{7}$ Geng et al. reported a case of an abdominal metastatic germ cell tumour with an unknown primary source. ${ }^{8}$ In such cases, it is important to exclude a primary tumour of the gonads, as was done in the present case. The role of radiology is very important to help localise the primary site of a yolk sac tumour. ${ }^{9,10}$ There may be an occult primary tumour in the testes with metastatic disease in the stomach and peritoneum. ${ }^{11}$

Although the role of radiation in treating the recurrence of primary mediastinal germ cell tumours is well established, little information is available regarding primary gastric yolk sac tumours. Despite recurrence after surgery and chemotherapy, Sakaguchi et al. reported the successful long-term survival of a patient with a yolk sac tumour after radiotherapy. ${ }^{12}$ In general, a multidisciplinary approach is important when treating malignancies at any tertiary care centre.

\section{Conclusion}

Primary gastric yolk sac tumours are extremely rare. An occult primary tumour in the gonads should be excluded radiologically before confirming a diagnosis. Furthermore, immunohistochemical analysis is vital, particularly with regards to AFP positivity.

\section{References}

1. Horwich A, Nicol D, Huddart R. Testicular germ cell tumours. BMJ 2013; 347:f5526. doi: 10.1136/bmj.f5526.

2. Guo YL, Zhang YL, Zhu JQ. Primary yolk sac tumor of the retroperitoneum: A case report and review of the literature. Oncol Lett 2014; 8:556-60. doi: 10.3892/ol.2014.2162.

3. Uematsu M, Yokouchi H, Tanino Y, Munakata M. Renin-producing germ cell tumor in the pineal apparatus and mediastinum: A rare case report. J Cancer Res Ther 2018; 14:S806-8. doi: 10.41 03/0973-1482.180682.

4. Büyükpamukçu M, Varan A, Küpeli S, Ekinci S, Yalcin S, Kale G, et al. Malignant sacrococcygeal germ cell tumors in children: A 30-year experience from a single institution. Tumori 2013; 99:51-6. doi: 10.1700/1248.13788.

5. Magni E, Sonzogni A, Zampino MG. Primary pure gastric yolk sac tumor. Rare Tumors 2010; 2:e10. doi: 10.4081/rt.2010.e10.

6. Suzuki T, Kimura N, Shizawa S, Yabuki N, Yamaki T, Sasano H, et al. Yolk sac tumor of the stomach with an adenocarcinomatous component: A case report with immunohistochemical analysis. Pathol Int 1999; 49:557-62. doi: 10.1046/j.1440-1827.1999.00907.x.

7. Satake N, Chikakiyo M, Yagi T, Suzuki Y, Hirose T. Gastric cancer with choriocarcinoma and yolk sac tumor components: Case report. Pathol Int 2011; 61:156-60. doi: 10.1111/j.1440-1827.20 10.02635.x.

8. Geng HL, Shi SS. [Clinicopathologic characteristics of primary gastric choriocarcinoma: Report of a case]. Zhonghua Bing Li Xue Za Zhi 2017; 46:571-2. doi: 10.3760/cma.j.issn.0529-5807. 2017.08.012.

9. Napaki S. Combined yolk sac tumour and adenocarcinoma of the oesophago-gastric junction. Pathology 2004; 36:589-92. doi: $10.1080 / 00313020400011003$.

10. Wang L, Tabbarah HJ, Gulati P, Rice S, French SW. Gastric adenocarcinoma with a yolk sac component: A case report and review of the literature. J Clin Gastroenterol 2000; 31:85-8.

11. Garcia RL, Ghali VS. Gastric choriocarcinoma and yolk sac tumor in a man: Observations about its possible origin. Hum Pathol 1985; 16:955-8. doi: 10.1016/S0046-8177(85)80137-4.

12. Sakaguchi M, Maebayashi T, Aizawa T, Ishibashi N, Fukushima S, Saito T. Successful radiotherapy in postoperative recurrence of a primary mediastinal yolk sac tumor: A case report. Thorac Cancer 2016; 7:358-62. doi: 10.1111/1759-7714.12302. 